\title{
Geschlossene Wohnkomplexe in Quito - Naturraum und rechtliche Rahmen- bedingungen als Einflussgrössen für Verbreitung und Typisierung
}

\section{Petra Kohler, Innsbruck}

\section{Einleitung}

Während in den Städten der westlichen Hemisphäre private, von der Außenwelt abgeschottete Wohnstätten über Jahrhunderte «uncommon places for uncommon people» waren (BLAKELY \& SNYDER 1997:4), ist die Suche nach der Abgeschiedenheit und Sicherheit hinter hohen Mauern heute zu einem der wichtigsten Ordnungsprinzipien der modernen Stadtentwicklung geworden.

In den Städten der USA haben immer tiefere soziale Gegensätze und die steigende Angst vor Gewalt und Kriminalität zu einer starken sozialräumlichen Segregation geführt, die sich dort in einer zunehmenden Fragmentierung des städtischen Raumes und der städtischen Gesellschaft äußert. Dabei haben sich zwei Pole herausgebildet: das unfreiwillig gebildete «Ghetto» der Ausgeschlossenen als Ort der «gefährlichen Klasse» am unteren Ende der städtischen Hierarchie und die freiwillig gewählte exklusive Enklave als Lifestyle und Prestige Community und Ort der Wohlhabenden an deren Spitze. Ausgehend von amerikanischen Vorbildern ist es auch in den lateinamerikanischen Metropolen spätestens seit den achtziger Jahren zur Ausbildung von ganzheitlich geplanten, umschlossenen Wohnvierteln mit aufwendigen Sicherheitsvorkehrungen gekommen, hinter deren Mauern die Angehörigen der Oberschicht und in der Folge auch der Mittelschicht das Chaos der Stadt zurücklassen und nach einer "privaten Idylle» suchen.

Obgleich sich das Phänomen der barrios cerrados (umzäunte Wohnformen) inzwischen in ganz Lateinamerika verbreitet hat und wichtige Einflüsse auf die Stadtstruktur und -entwicklung nach sich gezogen hat, wurde es in der öffentlichen Diskussion bisher kaum wahrgenommen. Generell, speziell aber in Lateinamerika selbst, stand man der Entwicklung lange Zeit abwartend oder «nicht erkennend» gegenüber, so dass die ersten Veröffentlichungen erst in den letzten Jahren entstanden und sich meist nur auf Fallstudien beschränken (BORSDORF 1998, 2000; MEYER \& Bähr 2001; Pöhler 1999; Coy \& Pöhler 2001; Suárez 1997; Janoschka 2000; Cabrales \& Canosa 2001).

Ziel dieses Beitrages ist es, einen Überblick über die Bedeutung, Entwicklung und Verbreitung der barrios cerrados in Quito zu geben, wobei besonders auf die Auswirkung der rechtlichen Rahmenbedingungen und der naturräumlichen Voraussetzungen für die Allokation der juristisch unterschiedlich definierten barrios cerrados eingegangen wird.

\section{Sozialräumliche Segregation, Verteilung und Bewertung der barrios cerrados in Quito}

Obwohl Quito bezüglich der Entwicklung der barrios cerrados eher zu den «Nachzüglern» in den lateinamerikanischen Metropolen zu zählen ist, haben die Auswirkungen der Transformation, Globalisierungstendenzen, vor allem aber massive innenpolitische Probleme im Laufe der Zeit zu erheblichen Abschottungstendenzen geführt, die sich inzwischen tief im Raumbild der Hauptstadt manifestiert haben.

Während Mitte der achtziger Jahre in anderen Großstädten Lateinamerikas der Trend zu barrios cerrados schon weit fortgeschritten war, präsentierte sich das Stadtbild von Quito noch weitgehend frei von abgeschlossenen Vierteln. Die negativen Auswirkungen der Transformationspolitik, wie die steigende Arbeitslosigkeit, ein Verfall der Reallöhne und das Abrutschen immer breiterer Bevölkerungsschichten unter die Armutsgrenze, gesteigerte Globalisierungstendenzen und ein durch innenpolitische Probleme hervorgerufenes Sicherheitsbedürfnis, führten in den folgenden Jahren aber auch in Quito zu einer Verschärfung der Segregationstendenzen, die sich inzwischen durch einen wahren Bauboom der barrios cerrados äußern.

Bedingt durch die spezielle naturräumliche Lage der Stadt und die genaue gesetzliche Regelung der umzäunten Wohnsiedlungen ist es zu einem spezifischen Verteilungsmuster der barrios cerrados in Quito gekommen, das nach der Diskussion der wichtigen physiognomischen und rechtlichen Parameter im Detail präsentiert wird.

\subsection{Die naturräumliche Ebene}

Schon seit jeher stand die physiognomisch bedingte Nord-Süd Ausdehnung Quitos einer modellhaften Entwicklung entgegen (vgl. Modell der lateinamerikanischen Stadt, BORSDORF 1982) und begünstigte die Entstehung des heutigen, klar segregierten Stadtbildes.

Heute präsentiert sich Quito in einer bandstadtähnlichen Form von $35 \mathrm{~km}$ Länge und nur fünf bis acht 
Kilometern Breite auf einem Niveau von 2800 m über dem Meeresspiegel. Während das Terrain im Osten zum benachbarten Tal steil abfällt, wird die Stadt im Westen von ihrem Hausberg, dem 4794 m hohen Pichincha, flankiert (Mena 2001:5).

Durch das koloniale Zentrum getrennt, haben die nördlichen und südlichen Stadtteile einen völlig unterschiedlichen Entwicklungsverlauf genommen und weisen klar voneinander getrennte räumliche und soziale Strukturen auf (BORSDORF \& STADEL 1997). Stark generalisierend könnte man feststellen, dass das gesamte südliche Stadtgebiet von Mittelklasse-, Unterschicht- und Marginalvierteln eingenommen wird, während der Norden und in jüngster Zeit auch die Täler östlich von Quito der Mittel- und Oberschicht vorbehalten sind. Während im Westen der Prozess der Marginalisierung an den Hängen des Pichinchas nicht mehr aufzuhalten ist, entwickelt sich die Besiedlung der östlichen Talgebiete genau in die Gegenrichtung. Die landschaftlichen Reize und klimatische Vorteile bewegen immer mehr Menschen aus der Stadt, hierher zu ziehen. Besonders die Gebiete des Valle de los Chillos und des Valle de Tumbaco verzeichneten im letzten Jahrzehnt einen großen Zuwachs an mittleren und gehobenen Bevölkerungsgruppen (SARVAN \& TEgeler 1999: 10).

$\mathrm{Da}$ jedoch keine eindeutige Planung, ja nicht einmal eine klar definierte Politik für diesen Raum existiert, unterliegt die Besiedlung der Region vornehmlich den Regeln der Marktwirtschaft. Als Konsequenz entsteht eine Suburbia, die neben der Verlagerung der Wohnfunktion auch die Konzentration wichtiger Funktionen der Stadt an den Stadtrand (Shopping Malls, Universität, Flughafen) mit sich brachte (MENA 2001:9).

\subsection{Die gesetzliche Ebene}

\section{Lateinamerika und rechtliche Aspekte}

«In vielen Ländern Lateinamerikas hat sich die verfassungsmäßige Rechtsordnung als irrelevant für die Legitimation der Handlungen der politischen Machthaber erwiesen» (GARZÓN VALDÉS 1994: 53).

Zwar sind auch in Europa und den restlichen «westlichen» Ländern immer wieder Divergenzen zwischen der offiziellen und der tatsächlich angewandten Rechtsordnung festzustellen, aber in keinem Teil der Welt sind sie so groß wie in Lateinamerika. Die Interpretation und in der Folge die Ausübung des Rechts basiert auf einer alten, in den Augen des westlichen Betrachters, paradoxen Tradition:Während einerseits ein enormes Interesse an den verschiedensten Gesetzestexten besteht und enormes Vertrauen in die Verfassung als wichtigstes Instrument der Regulierung des sozialen Lebens gelegt wird, scheint der Bruch zwischen
Rechtsordnung und sozialer Wirklichkeit jedoch kein Grund zur Beunruhigung zu sein. Dieses Phänomen wird häufig als «Pathologie des Rechts» bezeichnet.

«Analysiert man das lateinamerikanische Verfassungsrecht, dann muss man folglich immer ausdrücklich dazu sagen, ob man vom Buchstaben der Verfassung oder von ihrer tatsächlichen Wirklichkeit spricht» (Garzón Valdéz 1994: 45).

Dieser Grundsatz scheint auch für die gesetzliche Regelung der barrios cerrados in Ecuador zu gelten. Die folgenden Ausführungen werden bestätigen, dass zwischen der prinzipiellen gesetzlichen Regelung und Klassifikation der barrios cerrados und ihrer schlussendlichen Interpretation Divergenzen festzustellen sind, deren Auswirkungen sich auch im Raum zeigen.

Eine Analyse des ecuadorianischen Wohnbaugesetzes ergibt Folgendes: Prinzipiell gliedern sich umzäunte Wohnformen (barrios cerrados) in urbanizaciones cerradas (abgeschlossene Wohnviertel) und conjuntos cerrados (geschlossene Wohnblöcke). Ihre Differenzierung bezieht sich weniger auf die Ausstattung - beide Formen erfüllen alle genannten Anforderungen in Bezug auf Sicherheit und Infrastruktur - vielmehr unterscheiden sie sich durch unterschiedliche Erschließungsformen und gegensätzliche Besitz- beziehungsweise Rechtsverhältnisse, die sich schließlich in Determinanten wie Größe, Dichte und Baustil niederschlagen.

\section{Urbanización cerrada}

Das Registro Oficial Nº 310 (Organo del Gobierno DEL ECUADor 1998) definiert in Artikel II 29 die urbanización (cerrada) als ein Stadtviertel, in dem «das Gesamtterrain in mindestens zehn Parzellen unterteilt werden muss, deren Bebauung von den Besitzern selbst übernommen wird». Nach der Parzellierung der Fläche, der Umzäunung des Geländes und der Bereitstellung der nötigen Infrastruktureinrichtungen wie Strassen, Bürgersteige, Kanalisation, Wasser, Strom, Gas und Telefonleitungen durch den Bauträger werden die einzelnen Flächen verkauft und von den neuen Besitzern in Eigenregie bebaut.

Obwohl die meisten urbanizaciones ummauert und von Sicherheitsleuten bewacht sind, enthalten sie «öffentlich» zugängliche Grünflächen und Strassen. «Toda parcelación del suelo debe contemplar un sistema vial de uso público.» (Jede Aufteilung des Bodens muss über ein öffentlich zugängliches Straßensystem verfügen, Registro Oficial $\mathrm{N}^{\circ} 310$ : 7). Rechtlich gesehen sind alle Absperrungen unzulässig, da sämtliche Strassen und Wege öffentliches Eigentum darstellen und jedem Bürger das Recht vorbehalten ist, diese zu nützen. Die Stadtverwaltung scheint sich jedoch den Forderungen der mächtigen Immobilienfirmen zu beugen und beweist so, dass die öffentliche 


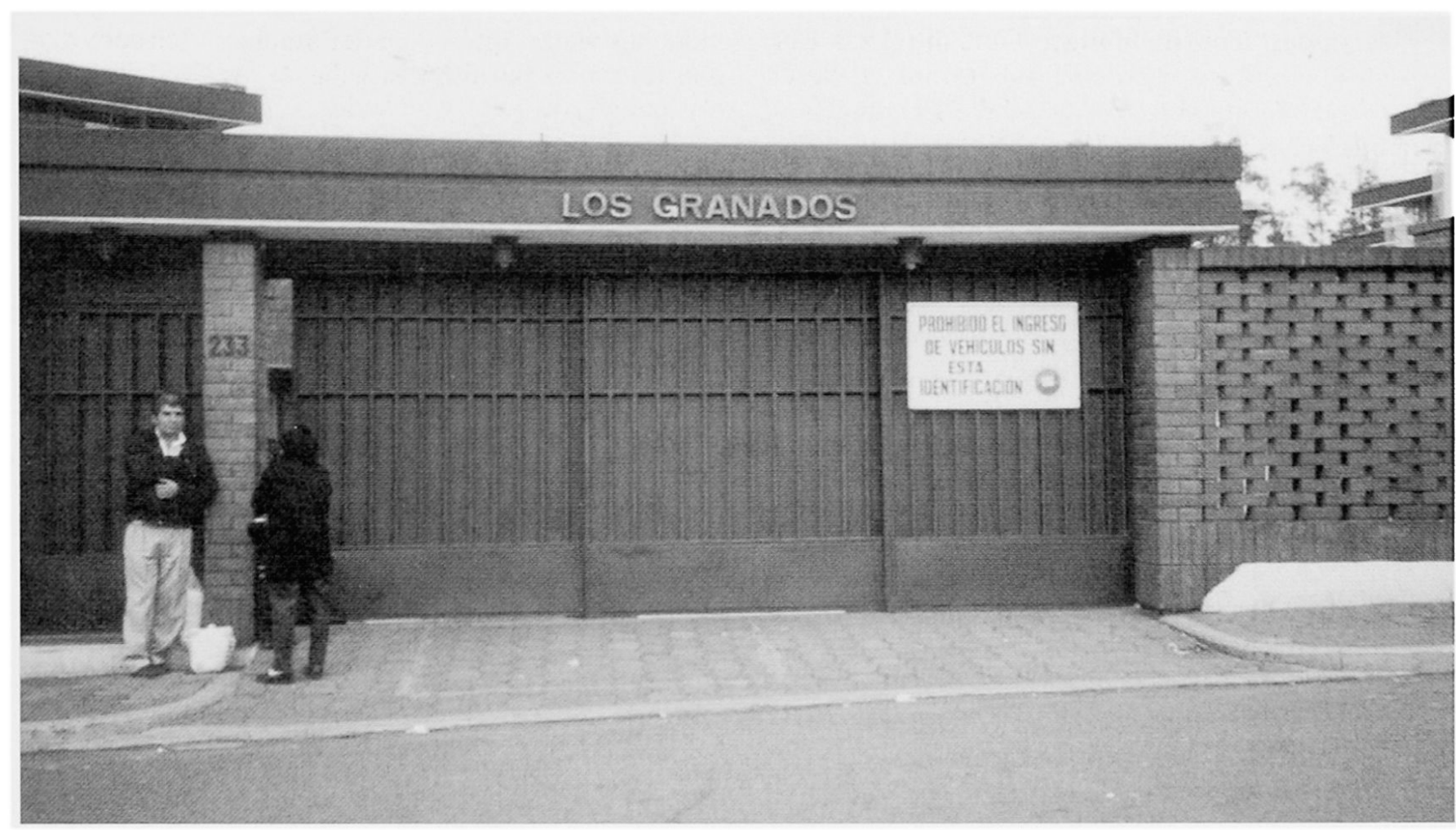

Foto 1: Haupteingang zum Mittelschicht-conjunto Los Granados in Quito

Foto: P. KOHLER

Main entrance to the middle class "conjunto" Los Granados in Quito

L'entrée principale du «conjunto» Los Granados, pour classes moyennes, à Quito

Regulierung immer mehr hinter die Interessen des privaten Kapitals zurückfällt, und es folglich auch im ecuadorianischen Gesetzessystem Divergenzen zwischen dem tatsächlichen Gesetzestext und seiner schlussendlichen Auslegung gibt. Bezüglich der urbanizaciones scheinen sich die Interessen von Staat und Privatwirtschaft in diesem Fall sogar zu ergänzen. Der Bauherr verpflichtet sich, alle Infrastruktureinrichtungen bereitzustellen und diese im Anschluss ohne Kosten an das Municipio zu übergeben.

\section{Conjunto cerrado}

Im Gegensatz zu den urbanizaciones cerradas entstehen conjuntos cerrados als ganzheitlich geplante Siedlungseinheiten von privaten Projektentwicklern. Nach der Parzellierung, Ummauerung und Bereitstellung der vorgeschriebenen Infrastruktureinrichtungen, Strassen und Grünflächen werden die Gebäude vom Bauträger selbst errichtet und anschließend verkauft (Registro Oficial $\mathrm{N}^{\circ}$ 310: 15). Wie bereits erwähnt, befinden sich prinzipiell alle Strassen und Wege im Besitz der öffentlichen Hand, außer es handelt sich um Zufahrtsstrassen und -wege zu Privatgrundstücken. Im Falle der conjuntos cerrados macht man sich diese Ausnahmeregelung zu Nutze und deklariert das gesamte Gelände als ein Grundstück mit Zufahrtswegen zu den einzelnen Parzellen. Im Unterschied zu den urbaniza- ciones cerradas, in denen man sich ausschließlich ein Teilgrundstück aneignen kann und sich die Strassen, Grünflächen und Infrastruktureinrichtungen eigentlich im Besitz des Staates befinden, erwirbt man in einem conjunto cerrado offiziell einen gewissen Prozentsatz am Gesamtgrundstück. Da sich also das gesamte Gebiet inklusive aller Gemeinschafts- und Infrastruktureinrichtungen im Besitz der Bewohner befindet, kann der Staat einer Umzäunung nichts entgegensetzen.

Im Gegenzug ist die Gemeinschaft der conjuntoBewohner für die Erhaltung und Wartung aller Infrastruktureinrichtungen und Gemeinschaftseinrichtungen verantwortlich. Im Gegensatz zu den urbanizaciones cerradas reichen schon zwei Einfamilienhäuser beziehungsweise Appartementhäuser mit entsprechender Grünfläche und Bewachung aus, um die Anlage als conjunto cerrado deklarieren zu können.

Eine weitere Form der Segregation ist das Absperren von Straßen oder Straßenzügen. Diese illegale Form der Selbstabgrenzung findet man vor allem im dicht bebauten Stadtgebiet, wo eine nachträgliche Ummauerung einzelner Häuser aus Platzgründen nicht möglich ist. Besonders in wohlhabenderen Vierteln, in denen die Bewohner über Einfluss auf die Stadtverwaltung 
oder zumindest über die nötigen Geldmittel zur Einflussnahme verfügen, werden Straßen einfach durch Ketten oder Zäune abgesperrt und die Eingänge von privatem Sicherheitspersonal bewacht.

Die speziellen gesetzlichen Voraussetzungen haben in Verbindung mit dem bereits erwähnten naturräumlichen Aspekt zur typischen Verbreitung der barrios cerrados in Quito geführt (Foto 1).

\subsection{Anzahl und räumliche Verteilung}

Es gibt im Großraum Quito viel weniger urbanizaciones als conjuntos. Die Mindestanzahl von zehn Parzellen erfordert eine bestimmte Gesamtgröße einer urbanización. Der dafür entstehende Flächenbedarf führt nahezu zwangsläufig zur Allokation solcher Anlagen am Stadtrand. Von den insgesamt 27 Mittel- und Oberschicht-urbanizaciones liegen 21 in der Suburbia, davon 19 im Großraum Tumbaco-Cumbaya. Auch die staatlich geförderten Projekte verlagern sich in zuneh- mendem Maße vom Süden der Stadt auf den suburbanen Raum im Bereich des Valle de los Chillos und in Calderon (Abb. 1).

Conjuntos haben zwar keine vorgeschriebene Größenbeschränkung, in den meisten Fällen bestehen sie jedoch aus weniger als zehn Parzellen und beschränken sich auf ein Minimum an Gemeinschaftseinrichtungen. Als wichtigstes Kriterium gilt der Sicherheitsaspekt. Die verschiedene Größenverteilung bedingt, dass conjuntos viel häufiger im Stadtgebiet vorzufinden sind als urbanizaciones. Der Bauboom der letzten Jahre bewirkte jedoch auch eine Zunahme der conjuntos im suburbanen Raum, wobei sich hierbei vor allem die Täler de Tumbaco und de los Chillos als Zentren herausgebildet haben.

Die Verteilungskarte zeigt, dass sich die Mehrzahl der seit 1997 errichteten conjuntos im Norden (68) und im suburbanen Raum (50) befinden. Im Süden der Stadt

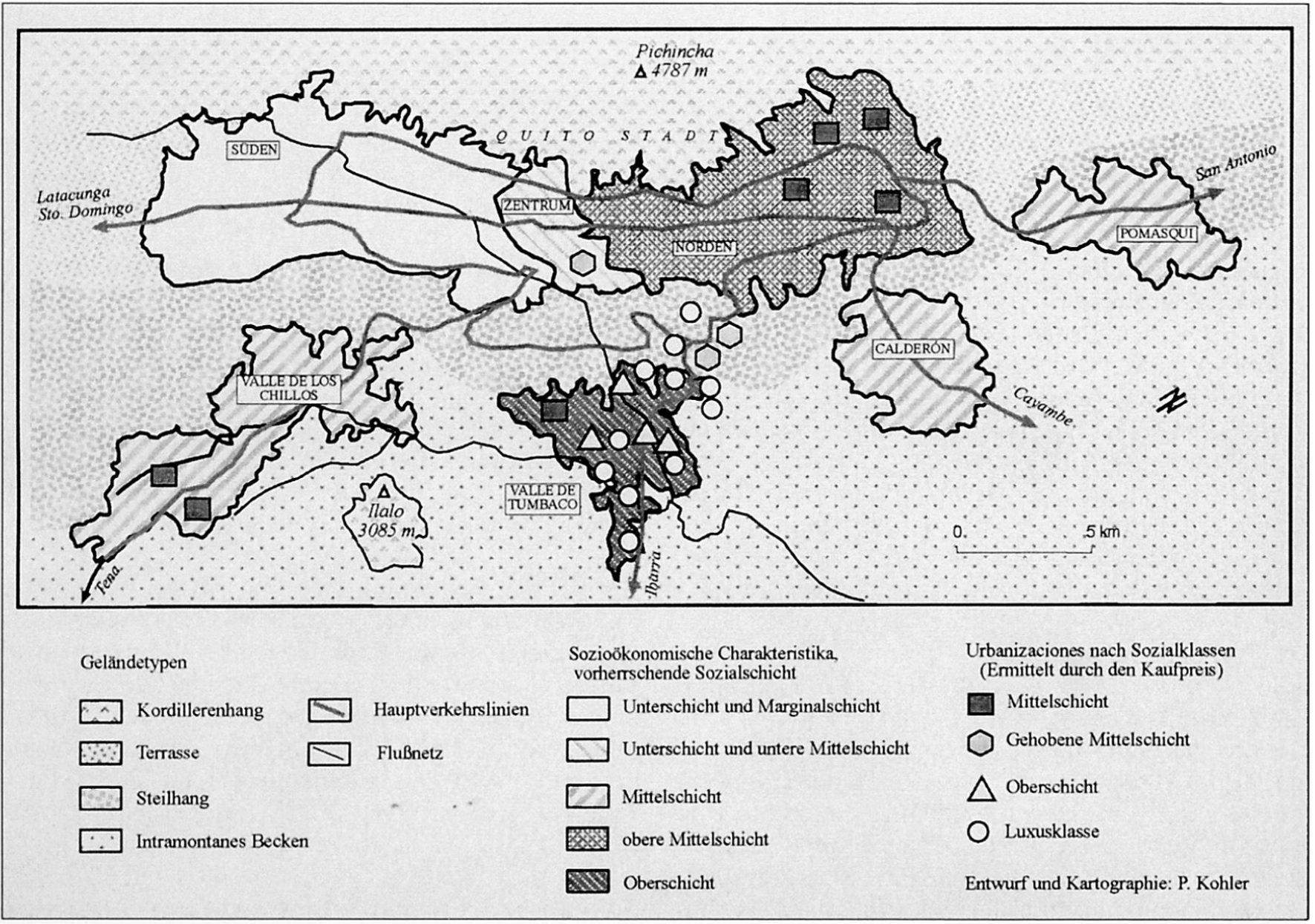

Abb. 1: Mittel- und Oberschicht-urbanizaciones im Grossraum Quito Middle class and upper class "urbanizaciones» in Greater Quito "Urbanizaciones», pour classes moyennes et supérieures, dans le Grand Quito Kartengrundlage: GomeZ, E. (2000): Guia Informativa de Quito; GRIDCON (Hrsg.) (2000): Mapa de Quito 


\begin{tabular}{|l|l|l|l|l|}
\hline Soziale Klasse & Zentrum & Norden & Süden & Suburban \\
\hline Mittelschicht & $2^{2}$ & $31^{21}$ & $9^{3}$ & $21^{4}$ \\
\hline Gehobene Mittelschicht & 0 & $26^{16}$ & $2^{0}$ & $21^{0}$ \\
\hline Oberschicht & 0 & $5^{2}$ & 0 & $4^{0}$ \\
\hline Gehobene Oberschicht & 0 & $6^{2}$ & 0 & $4^{0}$ \\
\hline
\end{tabular}

$\mathrm{n}^{\mathrm{x}}: \mathrm{n}=$ Gesamtzahl, $\mathrm{x}=$ Anzahl der Appartementhäuser an der Gesamtzahl

Definition der Sozialen Klasse nach GRIDCON 2000:

Mittelschicht $=$ US $\$ 17190-35392$ Kaufpreis/Wohneinheit

Gehobene Mittelschicht $=$ US\$35393 -70784 Kaufpreis/Wohneinheit

Oberschicht $=$ US $\$ 70785-111232$ Kaufpreis/Wohneinheit

Gehobene Oberschicht $=>$ US $\$ 111233$ Kaufpreis/Wohneinheit

Tab. 1: Verteilung der seit 1997 fertiggestellten conjuntos nach Sozialklassen Distribution of the "conjuntos» completed after 1997, according to social classes Répartition des "conjuntos», achevés après 1997, présentés par classes sociales Quelle: Sistema de Información Inmobiliaria Dinámica 1997-2000, GRIDCON

entstanden innerhalb der letzten drei Jahre elf neue Projekte der Mittelschicht, fünf weitere sind in Planung (GRIDCON 2001) (Abb. 2).

Ein wichtiger Unterscheidungspunkt besteht in der Bebauung der Fläche. Grundsätzlich ist bei beiden Formen der barrios cerrados eine klare Präferenz der Einfamilienhäuser festzustellen. Während sich conjuntos meist aus Appartements oder Einfamilienhäusern zusammensetzen und nur in den seltensten Fällen eine Mischung aus beiden Formen zu finden ist, weisen urbanizaciones meist eine heterogene Bebauung auf. Besonders in den Unterschicht- und Mittelschicht-urbanizaciones ist ein Nebeneinander von Einfamilien- und Appartementhäusern zu finden, die Anlagen der oberen Bevölkerungsschichten bestehen jedoch fast ausschließlich aus Einfamilienhäusern.

\subsection{Soziale Verteilung}

Die Untersuchung hat gezeigt, dass sich das Phänomen der barrios cerrados bis vor kurzem fast ausschließlich auf die Mittel- und Oberschicht konzentriert hat. Erst seit dem Aufkommen von staatlichen Unterstützungen wurde es auch für die untere Mittelklasse und die Unterschicht möglich, sich in geschlossenen Wohnsiedlungen zu organisieren. Neben den bisher üblichen Finanzierungshilfen gibt es seit Juli 1999 das vom Ministerio de Vivienda ins Leben gerufene Projekt «Bono para vivienda urbana nueva», das hauptsächlich junge, sozial bedürftige Familien beim Erwerb eines Hauses oder einer Wohnung unterstützen soll.
Während die Mehrzahl der conjuntos cerrados für die Mittelschicht konzipiert ist, handelt es sich bei den urbanizaciones eher um Wohnanlagen der gehobenen Gesellschaftsschichten.

Insgesamt wurden in Quito seit 1997131 conjuntos cerrados vermarktet (GRIDCON 2001), die zusammen über 7593 Wohneinheiten verfügen. Eine Analyse der Kaufpreise bestätigt, dass sich das Angebot immer stärker an die mittlere soziale Schicht richtet. Von den insgesamt 131 conjuntos wurden über $85 \%$ für die Mittelschicht und die gehobene Mittelschicht gebaut, lediglich 21,4\% der Angebote waren den Vertretern der Oberschicht zugedacht (vgl. Tab 1).

Zu Beginn des Jahres 2001 gab es im Großraum Quito 36 fertiggestellte urbanizaciones, fünf staatlich unterstützte Anlagen befanden sich in der Bauphase und sechs weitere sind noch in Planung. Dabei waren 13 Anlagen der Mittelschicht und gehobenen Mittelschicht und 16 der Oberschicht und Luxusklasse zuzuordnen, sieben Wohneinheiten entstanden durch staatliche Förderungen (GRIDCON 2001) (Tab. 2).

\section{Typologie der barrios cerrados im Großraum Quito}

\subsection{Urbanizaciones cerradas}

Während die Ausgangsidee der conjuntos cerrados das sichere Wohnen im Stadtgebiet war, entwickelten sich urbanizaciones cerradas ursprünglich aus dem Zusammenlegen und Umzäunen ehemaliger Wochen- 


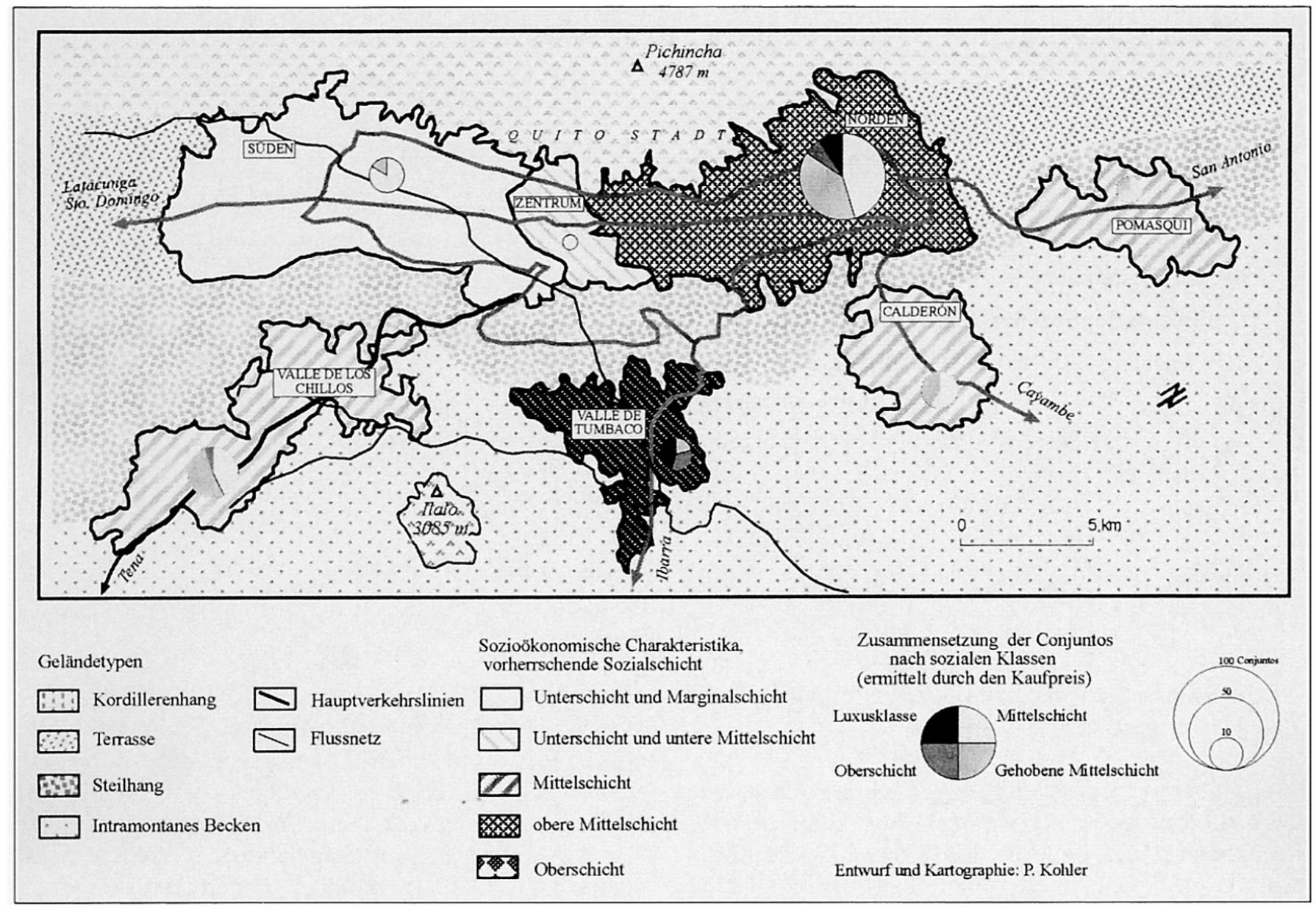

Abb. 2: Conjuntos im Grossraum Quito

"Conjuntos» in Greater Quito

"Conjuntos» dans le Grand Quito

Kartengrundlage: Gomez, E. (2000): Guia Informativa de Quito; GRIDCON (Hrsg.) (2000): Mapa de Quito

endhäuser im suburbanen Raum beziehungsweise aus Siedlungsanlagerungen an luxuriöse Freizeitclubs der Mittel- und Oberschicht am Stadtrand oder in den Tälern. Auf der Suche nach einer besseren Lebensqualität flüchteten wohlhabende Quiteños bereits Mitte der sechziger Jahre aus der immer lauter werdenden Stadt in die nahegelegenen Täler, so dass im Laufe der Zeit viele Wochenendhäuser zu festen Wohnsitzen umfunktioniert wurden. Häufig entschloss sich die Nachbarschaft zur Anlage von gemeinschaftlichen Tennis- oder Golfplätzen, deren Benutzung sich über die Mitgliedschaft in einem Club regelte. Die Grundidee der urbanizaciones lag also in der gemeinschaftlichen Nutzung bestimmter Einrichtungen, der Sicherheitsaspekt kam erst später hinzu.

Die Verwaltung und Organisation einer urbanización erfolgt in der Regel durch eine Asociación, die sich aus ehrenamtlich tätigen Bewohnern der Anlage zusammensetzt und durch einen Vorstand nach außen repräsentiert wird. Das anfangs nur zweitrangige Sicherheitsbedürfnis ist inzwischen zum wichtigsten Gesichtspunkt einer urbanización geworden. Heute kommt es in vielen Fällen innerhalb der ohnehin schon ummauerten bewachten Anlagen zu einer zusätzlichen Abschottung, indem die einzelnen Grundstücke nochmals ummauert und von privat angestellten Sicherheitsleuten bewacht werden (Foto 2). Im Extremfall werden zusätzlich einzelne Straßenzüge abgesperrt, die nur von den Bewohnern beziehungsweise deren Besuchern betreten werden dürfen.

In vielen Luxus-urbanizaciones, vor allem in den erst kürzlich erbauten Lifestyle-Anlagen, gibt es eine Vielzahl an unterschiedlichen Sport- und Freizeiteinrichtungen, wie Fitnessräume, Golf-, Reit- oder Tennisplätze, daneben finden sich Supermärkte, Friseure, Restaurants, Schulen oder Kindergärten, Wäschereien und Banken. Ein hotelartiger Service, wie er beispielsweise in den gated communities der Luxusklasse von Rio de 


\begin{tabular}{|l|c|c|}
\hline Soziale Klasse & $\begin{array}{l}\text { Anzahl der geschlossenen } \\
\text { Wohnblöcke (conjuntos) }\end{array}$ & Prozent \\
\hline Mittelschicht & 63 & $48,1 \%$ \\
\hline Gehobene Mittelschicht & 49 & $37,4 \%$ \\
\hline Oberschicht & 9 & $6,9 \%$ \\
\hline Gehobene Oberschicht & 10 & $7,6 \%$ \\
\hline Gesamt & 131 & $100,0 \%$ \\
\hline
\end{tabular}

Zur Definition der Sozialen Klassen vgl. Tabelle 1

Tab. 2: Anzahl der seit 1997 fertiggestellten conjuntos nach Sozialklassen Number of the "conjuntos" completed after 1997, according to social classes Nombre de "conjuntos", achevés depuis 1997, par catégories sociales Quelle: Sistema de Información Inmobiliaria Dinámica 1997-2000, GRIDCON

Janeiro angeboten wird, konnte in Quito jedoch bisher nicht beobachtet werden.

Die Typisierung der urbanizaciones cerradas im Großraum Quito soll neben einer Differenzierung der sozialen Klassen anhand der Kriterien «Bauform» und «Lage» erfolgen. Als Grundlage für die Erstellung dieser Klassifizierung dienten eigene Beobachtungen, Interviews und Fragebögen, die in den urbanizaciones El Condado (Mittelschicht), Jardines del Batán (gehobene Mittelschicht), Jacaranda (Oberschicht) und Altos del Valle (gehobene Oberschicht) verteilt wurden (siehe Tab. 1).

Es ergeben sich folgende Typen:

- Typ 1: Exklusive urbanizaciones cerradas der Oberschicht und Luxusklasse

- Typ 2: Urbanizaciones cerradas als Kopie der U.S.amerikanischen Lifestyle-Communities

- Typ 3: Urbanizaciones cerradas der Mittelschicht und gehobenen Mittelschicht

- Typ 4: Subventionierte urbanizaciones cerradas der unteren Mittelschicht und Unterschicht

Ad 1: Exklusive urbanizaciones der Oberschicht und Luxusklasse sind in den letzten Jahren hauptsächlich in den Talabschnitten Tumbaco und Cumbaya auf ehemals landwirtschaftlich genutzten Flächen entstanden. Die Anlagen sind zwischen 50 ha und 150 ha groß, sie verfügen jedoch über eine deutlich geringere Parzellenanzahl bei zugleich größerer Grundstücksfläche als vergleichbare urbanizaciones niedriger Sozialklassen. Das Zusammenleben basiert auf Anonymität. Die spärlichen Gemeinschaftseinrichtungen werden selten in Anspruch genommen, da die meisten Häuser einen eigenen Swimmingpool, einen Tennisplatz oder zumindest eine ausgedehnte Grünfläche besitzen. Die meisten Bewohner bevorzugen es, innerhalb der urbanización ein ruhiges, zurückgezogenes Leben mit wenig Kontakt zu den Nachbarn zu führen, seine Freizeit verbringt man lieber im außerhalb gelegenen Countryclub, um dort den sozialen Verpflichtungen nachzugehen und Freunde zu treffen. Einige urbanizaciones verfügen über eigene Clubs. Da man jedoch traditionellerweise Mitglied im Club seiner Eltern, Freunde, oder Arbeitskollegen ist und die Auswahl des Countryclubs nicht vom Wohnort abhängt, werden diese meist von Personen, die nicht in der Anlage wohnen, frequentiert.

Die individuell errichteten Einfamilienhäuser sind exklusiv ausgestattet und verfügen zumeist über einen eigenen Dienstbotentrakt, wo durchschnittlich zwei Hausbedienstete dauerhaft untergebracht sind. Jede Parzelle ist extra umzäunt und wird von einem eigenen Sicherheitsdienst bewacht. Neben diesen privaten Wachmännern gibt es auch einen von Seiten der urbanización engagierten Sicherheitsdienst, der neben der Bewachung des Haupteingangstores auch Patrouillen innerhalb der urbanización übernimmt. Die Straßen wirken meist wie ausgestorben. Man kommt und geht mit dem Auto, die Kinder spielen auf den eigenen Grundstücken, nur hin und wieder trifft man einen schwer bewaffneten Wachmann. Laut den Befragungsergebnissen ist der Hauptgrund, in einer exklusiven urbanización zu wohnen, das «sichere Leben in einer schönen Umgebung».

Ad 2: Urbanizaciones als Kopie der US-amerikanischen Lifestyle-Communities gibt es in der ecuadorianischen Hauptstadt erst seit Ende der neunziger Jahre. Während in vielen anderen lateinamerikani- 


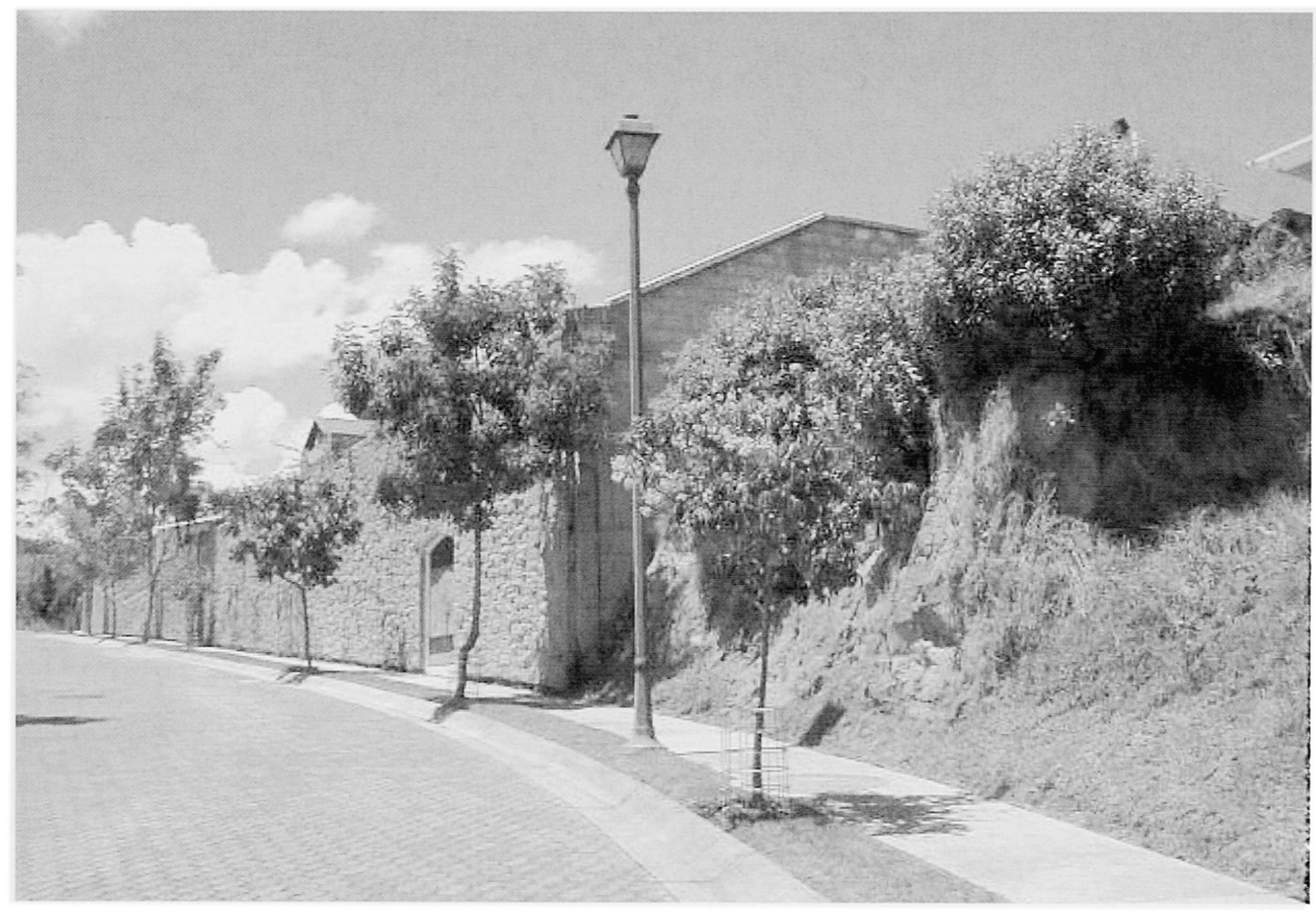

Foto 2: Mauern innerhalb der urbanización Altos del Valle in Quito Walls within the "urbanización" Altos del Valle in Quito

Foto: P. KOHLER Murs à l'intérieur de l'«urbanización» Altos del Valle en Quito

schen Städten der Trend zur Nachahmung des amerikanischen Lebensstils schon früher eingesetzt hat, manifestierte sich dieses Phänomen in Quito - zumindest im Immobiliensektor - erst in den letzten Jahren. Es war zwar schon lange modern, einzelne Häuser nach US-amerikanischem Vorbild zu konstruieren, die Planung von ganzen urbanizaciones in diesem Stil begann aber erst vor kurzem. Momentan stehen die zwei «Paradeprojekte», die urbanización Club Rancho San Francisco und die urbanización Arrayanes kurz vor ihrer Fertigstellung.

Die Anlagen befinden sich im suburbanen Raum und verfügen über eine exklusive Ausstattung, wobei die Freizeiteinrichtungen und Grünflächen teilweise überproportioniert erscheinen. Im Mittelpunkt der durchschnittlich zwischen 150 ha und 200 ha großen Anlagen befindet sich meist ein Countryclub, der von üppigen Gärten und Freizeiteinrichtungen und in weiterer Folge von den einzelnen Grundstücken, deren Flächen sich zwischen $1000 \mathrm{~m}^{2}$ und $5000 \mathrm{~m}^{2}$ bewegen, umgeben ist. Die Villen werden individuell errichtet und verfügen teilweise über Dienstbotentrakte. Die Freizeitmöglichkeiten sind weit gestreut. Außer den großen Golf- und Reitplätzen gibt es viele kleinere Plätze und Hallen für Tennis, Squash, Basketball, Volleyball oder Racketball. Außerdem stehen den Bewohnern neben einer Vielzahl an entspannungsfördernden Einrichtungen wie Saunen, Dampfbäder, Whirlpools, Massagesalons oder große Schwimmbäder eigens engagierte Fitnesstrainer zu Verfügung, die auf Wunsch individuell abgestimmte Fitnessprogramme entwikkeln und betreuen.

Im Gegensatz zu den exklusiven urbanizaciones der Oberschicht und Luxusklasse sind zwar viele Häuser privat bewacht, aber nicht immer ummauert. Durch eine hermetische Abriegelung der Anlage nach außen und einen gut organisierten Patrouillendienst im Inneren versucht man den Bewohnern das Gefühl von äußerster Sicherheit zu vermitteln und sie dadurch zu einer gemeinschaftsfördernden offenen Bauweise zu motivieren. Die Einrichtung der urbanización ist darauf ausgerichtet, dass die Bewohner die Anlage so 


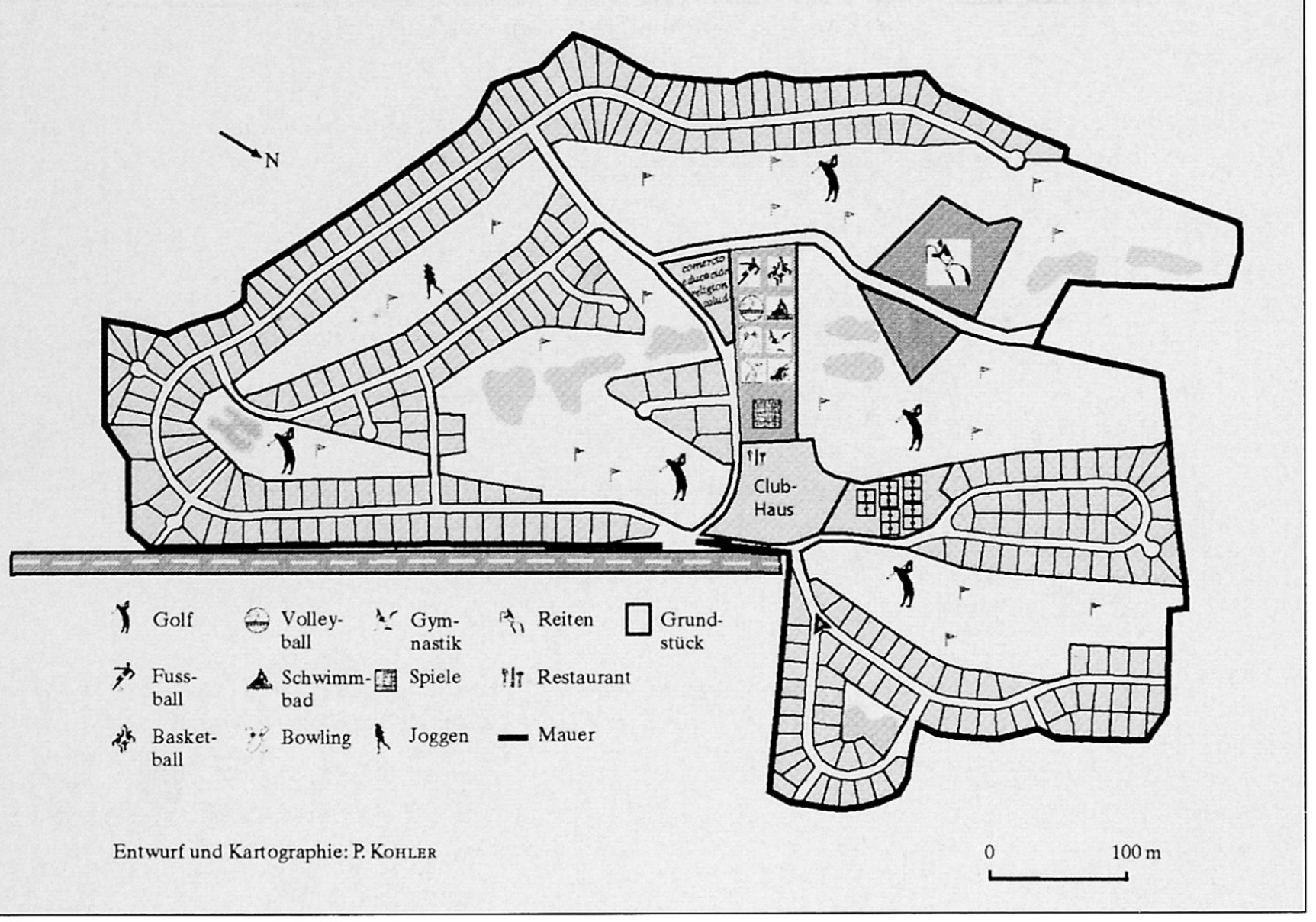

Abb. 3: Skizze der Lifestyle-urbanización Arrayanes

Sketch of the lifestyle-«urbanización» Arrayanes

Esquisse de l'«urbanización» lifestyle Arrayanes

Kartengrundlage: Dirección Club ArRayanes (Hrsg.) (1999): Informationsmaterial

wenig wie möglich verlassen müssen. Seine Freizeit verbringt man im eigenen Countryclub, meistens gibt es ein kleines Shopping Center, eine Bank, Friseure, einen Kindergarten und sogar eine Krankenstation. Anders als im erstgenannten Typ läuft alles darauf hinaus, sein Leben aktiv im Club zu verbringen. Sehen und gesehen werden, so lautet die Devise (Abb. 3).

Ad 3: Die urbanizaciones der Mittelschicht und gehobenen Mittelschicht befinden sich im Gegensatz zu den beiden erstgenannten Typen nicht ausschließlich im suburbanen Raum, sondern auch im Norden des Stadtgebietes. Die Bebauung ist geschlossener und besteht meist aus einer Mischung von Einfamilien- und zwei bis dreistöckigen Appartementhäusern. Zwar ist die urbanización umzäunt oder ummauert und mit einem bewachten Eingangstor versehen, die Abriegelung nach außen erfolgt jedoch nicht so konsequent wie in den Anlagen der Oberschicht. So kann es durchaus vorkommen, dass der Zutritt stellenweise nur durch einen Graben oder Stacheldraht verhindert wird. Oder es ist möglich, die Anlage über einen nicht bewachten Hintereingang zu betreten. Die Häuser innerhalb der urbanización sind nur teilweise umzäunt. Gesamtgröße, Bebauungsdichte und Parzellengröße variieren, und auch im Bezug auf die Exklusivität der Häuser sind innerhalb einer Anlage große Unterschiede festzustellen. Dem Erscheinungsbild fehlt eine gewisse Homogenität, meist ist eine Mischung der Baustile, Materialien, Farben und Größen zu beobachten. Es gibt gemeinsame Grünflächen, Spielplätze oder Sportanlagen. In den urbanizaciones der gehobenen Mittelschicht sind auch gemeinsame Einrichtungen wie Fitnessräume, Saunen, Dampfbäder oder ein Clubhaus vorhanden. Der Hauptunterschied zwischen Anlagen der Mittelschicht und gehobenen Mittelschicht liegt neben dem Kaufpreis nicht so sehr in der Ausstattung, sondern ist vielmehr gekennzeichnet durch ihre Lage. Während sich alle Anlagen der gehobenen Mittelschicht mit einer einzigen Ausnahme 


\section{geschlossene Wohnblöcke (conjuntos)}

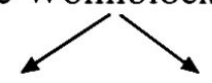

geschlossene Wohnblöcke mit Einzel- oder/

geschlossene Wohnblöcke mit und Reihenhäusern (conjuntos de casas)

Hochhäusern (conjuntos de edificios)

1. Conjuntos der Luxusklasse und Oberschicht

2. Conjuntos der gehobenen Mittelschicht und Mittelschicht

3. Condominios (gated communities) der Mittelschicht

4. Subventionierte conjuntos und condominios der Unterschicht

Abb. 4: Klassifikation der conjuntos cerrados im Grossraum Quito

Classification of the "conjuntos cerrados» in Greater Quito

Classification des "conjuntos cerrados» dans le Grand Quito

im «Nobelgebiet» Tumbaco-Cumbaya befinden, gibt es hier nur eine urbanización der Mittelschicht. Alle anderen befinden sich entweder im Norden der Stadt oder im Bereich des Valle de los Chillos.

Ad 4: Durch staatlich subventionierte urbanizaciones soll auch der unteren Mittelschicht und der Unterschicht der Erwerb oder Bau eines eigenen Hauses in einer sicheren Umgebung ermöglicht werden. Das Erscheinungsbild solcher urbanizaciones ist sehr bescheiden, die Häuser sind klein, einfach gebaut und ähneln einander sehr. Bis auf eine Grünfläche und vielleicht einen Kinderspielplatz gibt es keine Gemeinschaftseinrichtungen. Die Anlage ist zwar umzäunt, es gibt jedoch kaum Wachen, und der Eingang ist meist offen oder im besten Fall mit einer automatischen Schranke versehen. Der Kaufpreis für einen Quadratmeter Baugrund liegt zwischen 2 US\$ und 10 US\$. Neben der traditionellen staatlichen Kreditvergünstigung für den Erwerb oder Bau einer Immobilie gibt es auch für urbanizaciones das 1999 ins Leben gerufene staatliche Projekt «Bono para vivir», das vor allem jungen Familien eine Starthilfe beim Erwerb eines Eigenheimes bieten soll. Die Komplexe befinden sich im Süden und Norden des Stadtgebietes sowie im Valle de los Chillos und Calderón.

\subsection{Conjuntos cerrados}

Conjuntos cerrados treten im Gegensatz zu den urbanizaciones cerradas eher in der Innenstadt in Form von kleineren ummauerten Wohneinheiten oder Appartementhäusern oder mehr oder weniger einheitlichen Einfamilienhäusern mit einigen gemeinsamen Freizeiteinrichtungen auf. Vor allem im Stadtgebiet versucht man die Freizeiteinrichtungen in der Anlage zu reduzieren, so dass die meisten conjuntos neben einer gemeinsamen Grünfläche nur über einen offe- nen Sportplatz und vielleicht einen Versammlungsraum verfügen. Anders als in vielen urbanizaciones, wo sich das Leben so weit wie möglich innerhalb der Anlage abspielt, beschränken sich die Ansprüche der meisten conjunto-Bewohner auf ein sicheres Wohnen, die Freizeit wird lieber außerhalb verbracht.

Die ersten Anlagen entstanden in etwa zeitgleich mit den ersten Formen der urbanizaciones zu Beginn der achtziger Jahre in einem der damals nobelsten Wohngebiete, dem Viertel Gonzáles Suárez. Während die neue Wohnform am Anfang eher auf Skepsis stieß, ist seit den neunziger Jahren eine rege Bautätigkeit der ummauerten Wohnsiedlungen zu verzeichnen, so dass sich heute rund 1000 conjuntos im Großraum Quito befinden (Mena 2001; Kohler 2001) (Abb. 4).

\section{Fazit}

Die Entstehung und Verbreitung von barrios cerrados ist in den letzten Jahren zu einem der wichtigsten Bestandteile der dynamischen Veränderungsprozesse in lateinamerikanischen Städten geworden. Dieser Trend hat sich inzwischen auch in der ecuadorianischen Hauptstadt Quito durchgesetzt. Trotzdem scheint die Entwicklung noch nicht die Ausmaße vieler anderer lateinamerikanischer Großstädte erreicht zu haben. In der Innenstadt treten die umschlossenen Wohnsiedlungen meist in Form von conjuntos cerrados, kleineren ummauerten Wohneinheiten aus Appartementhäusern oder mehr oder weniger einheitlichen Einfamilienhäusern mit einigen gemeinsamen Freizeiteinrichtungen auf. Im Gegensatz dazu herrschen im randstädtisch-suburbanen Bereich urbanizaciones cerradas, großflächige Villenviertel mit aufwen- 
diger Infrastruktur und vielen Freizeiteinrichtungen, vor. Obwohl die Entwicklung des Siedlungsausbaus in den Talabschnitten Tumbaco und Cumbaya genau in diese Richtung geht, kam es in Quito bis heute zu keiner geschlossenen Ausbreitung des Phänomens wie beispielsweise im Barra da Tijuca in Rio de Janeiro, in dem rund 100.000 Menschen in geschlossenen Wohnanlagen leben (PÖHLER 1999: 8), oder in dem als «Dorfstadt» geplanten Projekt Nordelta in Buenos Aires, das 150.000 Menschen Wohnraum bieten sollte. Letzteres besitzt, neben den üblichen Infrastruktureinrichtungen wie Grünflächen und Sportplätze, Privatschulen, eine Berufsakademie, eine Privatuniversität, Einkaufszentren, Unterhaltungszentren, einen Bahnanschluss mit Expresszügen, eine direkte Autobahnanbindung und vieles mehr (JANOSCHKA 2002).

Es scheint alles darauf hinzuweisen, dass es sich bei der «neuen Generation» der barrios cerrados nicht mehr ausschließlich um Wohnviertel mit hochentwickelten Sicherheitseinrichtungen und gemeinsamen Freizeiteinrichtungen handelt. Durch eine Ausstattung der Anlagen mit umfassenden Versorgungs-, Bildungs- und Freizeiteinrichtungen wird es möglich, dem Chaos der Stadt zu entfliehen und sein Leben in einer selbstgeschaffenen heilen, sozial homogenen Welt zu verbringen. COY \& PöHLER (2001: 11) bezeichnen diese Form der freiwilligen Segregation als «selbstgewählte Ghettoisierung», die zu einer "Gefangenschaft in einer heilen Welt» führen kann.

Die bisherigen Untersuchungsergebnisse haben gezeigt, dass sich die barrios cerrados in Quito nur teilweise den globalen Prämissen anpassen und durchaus an spezifische soziökonomische Faktoren oder gesetzliche Strukturen angepasst sind. Fakt ist, dass sich auch hier der Trend zu abgeschlossenen Wohngebieten verstärkt und sich in den nächsten Jahren wohl auf den gesamten suburbanen Raum ausbreiten wird. Ob diese Entwicklung die angedeuteten Ausmaße erreichen wird oder ob die ecuadorianische Landeshauptstadt auch diesmal einen gesonderten Weg gehen wird, ist bis heute unklar und wird sich erst in nächster Zukunft erweisen.

\section{Dank}

Diese Arbeit wurde finanziert durch den Fonds zur Förderung wissenschaftlicher Forschung (Nummer P 14883).

\section{Literatur}

Blakely, E. \& M.G. SNyder (1997): Fortress America: Gated Communities in the United States. - Lincoln Institute of Land Policy, Cambridge.

BorSDORF, A. (1982): Die lateinamerikanische Gross- stadt. Zwischenbericht zur Diskussion um ein Modell. - Geographische Rundschau 34, 11: 498-501.

Borsdorf, A. \& C. Stadel (Hrsg.) (1997): Ecuador in Profilen. Landeskundliche Beobachtungen auf einer geographischen Exkursion. - = Inngeo 3, Innsbruck.

BorsDorf, A. (1998): Vom Casco Colonial zum Barrio Amurallado: Wohnformen in lateinamerikanischen Städten. - In: Kaller-DieTrich, M. (Hrsg.): Recht auf Entwicklung? Atención! Jahrbuch des Österreichischen Lateinamerika-Instituts 1. - Frankfurt a.M.: Südwind: 81-105.

Borsdorf, A. (2000): Condominios in Santiago de Chile als Beispiele sozialräumlicher Segregationstendenzen von Ober- und Mittelschicht in lateinamerikanischen Städten. - In: Peripherie, Zeitschrift für Politik und Ökonomie in der Dritten Welt 20, 80:25-40.

Cabrales Barajas, L.F. \& E. Canosa Zamora (2001): Segregación residencial y fragmentación urbana: los fraccionamientos cerrados en Guadalajara. - In: Espiral, Estudios sobre Estado y Sociedad Guadalajara 7: 223-253.

Coy, M. \& M. PöHLer (2001): Wohnghettos der Privilegierten. Die Fragmentierung der brasilianischen Stadt. Fallbeispiele aus Rio de Janeiro und Sao Paulo. - In: Matices 29: 123-138.

GARZON VALDES, E. (1994): Verfassung und Stabilität in Lateinamerika. - In: Borsdorf, A. (Hrsg.): Lateinamerika. Krise ohne Ende? - = Innsbrucker Geographische Studien 21: 43-61.

GRIDCON (Hrsg.) (1997-2000): Sistema de Información Inmobiliaria Dinámica (SIID). - Quito.

GRIDCON (Hrsg.) (2001): Características de la Demanda de Vivienda en Quito. - Quito.

Janoschra, M. (2000): Reich und arm in Buenos Aires. Barrios privados als neue Form der Suburbanisierung. - In: Praxis Geographie 30, 12: 60-62.

JanosChKa, M. (2002): Wohlstand hinter Mauern. Private Urbanisierungen in Buenos Aires. - = ISR-Forschungsbericht 28, Wien: Verlag der Österreichischen Akademie der Wissenschaften (im Druck).

Kanitscheider, S. (2002): Condominios und fraccionamientos cerrados in Mexiko-Stadt: Sozialräumliche Segregation am Beispiel abgesperrter Wohnviertel. In: Geographica Helvetica 57, 4: 253-263.

KoHLER, P. (2001): Sozialräumliche Segregation in lateinamerikanischen Städten am Beispiel der barrios cerrados in Quito, Ecuador. - Unveröffentlichte Diplomarbeit, Geographisches Institut, Universität Innsbruck. Mena, P. (2001): Environment and human security in local and regional national government goals. Quito: Municipio del Distrito Metropolitano de Quito, Dirección General de Planificación.

MeYer, K. \& J. BÄHR (2001): Condominios in Greater Santiago de Chile and their Impact on the Urban Structure. - Die Erde 132, 3: 293-321.

Organo del Gobierno del Ecuador (1998): Registro Oficial $N^{\circ} 310 .-$ Quito. 
PöHLER, M. (1999):Zwischen Luxus-Ghettos und Favelas. Stadterweiterungsprozesse und sozialräumliche Segregation in Rio de Janeiro. Das Fallbeispiel Barra da Tijuca. $-=$ Kleinere Arbeiten aus dem Geographischen Institut der Universität Tübingen 21, Tübingen. Sarvan, E. \& M. Tegeler (1999): Zukunftsfähige Siedlungsstrukturen für die Hänge des Pichincha. Unveröffentlichte Diplomarbeit, Universität Kaiserslautern, Institut für computergestützte Planungs- und Entwurfsmethoden (CPE) und TU München, Fachbereich Architektur und Städtebau.

SuAREZ, F. (1997): Nuevas tendencias residenciales en la Ciudad de Buenos Aires. - Carta Económica Regional 52, Instituto de Estudios Económicos y Regionales, Universidad de Guadalajara, 31-38.

\section{Zusammenfassung: Geschlossene Wohnkomplexe in Quito - Naturraum und rechtliche Rahmenbedingun- gen als Einflussgrössen für Verbreitung und Typisie- rung}

Die Entstehung und Verbreitung von barrios cerrados ist in den letzten Jahren zu einer der wichtigsten Ausdrucksformen des Transformationsprozesses in lateinamerikanischen Städten geworden. Bedingt durch Wirtschaftsreformen und die wachsende Einflussnahme der Globalisierung kam es in den meisten Metropolen schon früh zu einer Verstärkung der sozioökonomischen Disparitäten, welche sich inzwischen auch in Quito in einer scharfen sozialräumlichen Segregation äußern. Spezifische sozioökonomische, naturräumliche und gesetzliche Gegebenheiten haben seit jeher zu einem typischen zeitlich-räumlichen Schema der geschlossenen Wohnsiedlungen in der Stadt geführt. Heute erleben die nördlichen Stadtränder und die in östlicher Richtung anschließenden suburbanen Talgebiete einen bislang ungekannten Bauboom der barrios cerrados, der sich aber bis heute auf klar begrenzte, fragmentierte Räume beschränkt und noch keine zusammenhängende Regionen bildet, wie es etwa in Rio de Janeiro oder in Buenos Aires festzustellen ist.

\section{Summary: Enclosed Residential Complexes in Quito - natural and statutory realities influence distribution and form}

The development and dispersal of barrios cerrados has become one of the most important expressions of the transformation process in Latin American cities. Due to economic reforms and the growing influence of globalisation, socio-economic disparities began to show early in most metropolitan areas. In Quito too, these disparities have come to the fore in recent years, expressed by the formation of a sharp socio-spatial segregation. Specific socio-economic, natural and statutory realities have always led to a typical temporal and spatial pattern of closed urban residential areas.
Today the city's northern fringes and the suburban valley areas in the east are experiencing an unprecedented boom in the construction of barrios cerrados. These do not form contiguous areas as seen in other cities such as Rio de Janeiro or Buenos Aires, but rather they are scattered throughout Quito and characterised by obvious boundaries.

\section{Résumé: Complexes résidentiels fermés à Quito - Espace naturel et cadre juridique en tant qu'appuis de diffusion et de typisation}

La genèse et la diffusion de barrios cerrados sont devenues l'une des formes d'expression les plus importantes du processus de transformation des villes latinoaméricaines. Les réformes économiques et l'influence croissante de la mondialisation ont déjà renforcé relativement tôt les disparités socio-économiques dans la plupart des métropoles. Ces disparités se sont traduites entre-temps également à Quito par une forte ségrégation socio-spatiale. Des données socio-économiques, naturelles et légales y ont conduit dès l'origine à un schéma typique de lotissements résidentiels fermés au regard des rapports entre l'espace et le temps. A présent les franges urbaines septentionales et les vallées suburbaines adjacentes en direction de l'Est font l'objet d'un boom immobilier inédit dans le domaine des barrios cerrados. Ce boom se limite toutefois jusqu'à présent à des fragments d'espace clairement circonscrits. Il ne conduit pas encore à la formation de régions d'un seul tenant, comme s'est le cas par exemple à Rio de Janeiro ou à Buenos Aires.

\section{Didaktische Hinweise}

- Worin unterscheiden sich Ghettos von barrios cerrados?

- Inwiefern beeinflussen Naturraum und gesetzliche Vorgaben die Ausbildung von barrios cerrados in Quito?

- Wie lassen sich geschlossene Siedlungen im Grossraum Quito typologisieren und charakterisieren?

Mag. Petra Kohler, Institut für Stadt- und Regionalforschung. Österreichische Akademie der Wissenschaften, Postgasse 7/4/2, A-1010 Wien.

e-mail:Petra.Kohler@uibk.ac.at

\section{Manuskripteingang/received/manuscrit entré le} 11.9.2002

Annahme zum Druck/accepted for publication/accepté pour l'impression: 4.12 .2002 